\title{
Ocorrência de sinais e sintomas de disfunção temporomandibular em músicos
}

\section{Occurrence of signs and symptoms of temporomandibular dysfunction in musicians}

\author{
José Stechman Netoํㅜ Claudyane de Almeida ${ }^{2}$, Eliete Rodrigues Bradasch ${ }^{3}$, Lílian Cássia Bornia Jacob Corteletti ${ }^{4}$, \\ Kelly Cristina Silvério ${ }^{5}$, Morganna Maria de Aguiar Pontes ${ }^{6}$, Jair Mendes Marques ${ }^{7}$
}

\begin{abstract}
RESUMO
Objetivo: Verificar a prevalência de sinais e sintomas de disfunção temporomandibular em grupos de músicos profissionais, intérpretes de instrumentos de sopro e de cordas. Métodos: Participaram 92 músicos, com idades entre 18 e 58 anos. Foram entrevistados 70 músicos intérpretes de instrumentos de sopro (76,08\%) e 22 músicos intérpretes de instrumentos de cordas (23,91\%) pertencentes à Orquestra Sinfônica do Paraná, à Banda da Polícia Militar do Paraná ou à Banda do Exército. Foi realizada entrevista que constou de questões referentes à identificação, tempo de prática do instrumento e presença de sinais, sintomas e hábitos relacionados às disfunções temporomandibulares. Comparou-se as respostas dos músicos por meio do teste de diferença de proporções. Resultados: Trinta e nove músicos (42,3\%) percebem que rangem e/ou apertam os dentes, 23 (25\%) reportaram sentir dor na articulação temporomandibular, 39 músicos (42\%) relataram escutar ruídos na articulação temporomandibular, 37 (40\%) sensação de plenitude auricular e 33 (35\%) presença de zumbido. Não houve diferença significativa entre os instrumentistas de sopro e de corda no que se refere à presença de sinais e sintomas de disfunção temporomandibular. Conclusão: A somatória dos fatores apresentados coloca os praticantes de determinados instrumentos musicais como um grupo suscetível a apresentar sinais e sintomas de disfunção temporomandibular, incluindo sintomas auditivos, podendo tal prática ser considerada tanto um fator desencadeante, quanto um fator agravante ou perpetuador de um problema já existente.
\end{abstract}

Descritores: Articulação temporomandibular/patologia; Músculos mastigatórios; Música; Doenças profissionais/diagnóstico

Trabalho realizado na Universidade Tuiuti do Paraná - UTP - Curitiba (PR), Brasil.

(1) Pós-graduando (Doutorado) do Programa de Pos-graduação em Distúrbios da Comunicação da Faculdade de Ciências Biológicas e da Saúde da Universidade Tuiuti do Paraná - UTP - Curitiba (PR), Brasil; Professor adjunto da Universidade Tuiuti do Paraná - UTP - Curitiba (PR), Brasil

(2) Especialista em Disfunção Temporomandibular e Dor Orofacial pela Universidade Tuiuti do Paraná; Professora convidada do Curso de Especialização em Disfunção Temporomandibular e Dor Orofacial da Universidade Tuiuti do Paraná - UTP - Curitiba (PR), Brasil.

(3) Especialista em Disfunção Temporomandibular e Dor Orofacial pelo Conselho Federal de Odontologia; Professora assistente do Curso de Especialização em Disfunção Temporomandibular e Dor Orofacial da Universidade Tuiuti do Paraná - UTP - Curitiba (PR), Brasil.

(4) Doutora, Professora do Programa de Pos-graduação em Distúrbios da Comunicação da Faculdade de Ciências Biológicas e da Saúde da Universidade Tuiuti do Paraná - UTP - Curitiba (PR), Brasil.

(5) Doutora, Professora do Programa de Pos-graduação em Distúrbios da Comunicação da Faculdade de Ciências Biológicas e da Saúde da Universidade Tuiuti do Paraná - UTP - Curitiba (PR), Brasil.

(6) Pós-graduanda (Mestrado) do Programa de Pos-graduação em Distúrbios da Comunicação da Faculdade de Ciências Biológicas e da Saúde da Universidade Tuiuti do Paraná - UTP - Curitiba (PR), Brasil.

(7) Doutor, Professor do Programa de Pos-graduação em Distúrbios da Comunicação da Faculdade de Ciências Biológicas e da Saúde da Universidade Tuiuti do Paraná - UTP - Curitiba (PR), Brasil.

Endereço para correspondência: José Stechman Neto. R. Padre Anchieta, 1846/13, Curitiba (PR), Brasil, CEP: 80730-000.E-mail: stechman@onda.com.br Recebido em: 28/7/2008; Aceito em: 21/1/2009

\section{INTRODUÇÃO}

A música é um meio de expressar emoções e tem sido parte integrante das experiências do ser humano. A música está presente no cotidiano e, muitas vezes, atua como um marcador para as fases da nossa vida.

Quando se pensa em música, geralmente vem à mente a idéia de diversão e bem estar, e isto, talvez, proporcione certa dificuldade em considerar a execução de determinados instrumentos musicais como uma atividade capaz de trazer riscos para a saúde ${ }^{(1)}$

Nesta perspectiva, alguns estudos apontaram esta atividade como causadora de danos ocupacionais, especialmente, problemas músculo-esqueléticos relacionados a disfunções temporomandibulares e alterações auditivas ${ }^{(2-7)}$. Esses danos podem levar ao aparecimento de problemas de saúde capazes de interferir significativamente na habilidade e na atuação do músico, podendo, até mesmo, por fim à carreira do indivíduo.

Cabe colocar que a disfunção temporomandibular é um distúrbio geralmente relacionado ao estresse, que induz ao aumento da atividade muscular, frequentemente associada a hábitos parafuncionais, resultando em fadiga, espasmo e dor. O fator etiológico mais comum é a hiperatividade muscular, 
causada por má oclusão, estresse e/ou alterações posturais ${ }^{(8)}$.

Diariamente, muitos músicos praticam seus instrumentos por longos períodos. Algumas posições, pouco ergonômicas, são requeridas para manter o instrumento em posição durante a prática ${ }^{(9)}$.

No caso do violino e da viola, a pressão exigida para manter o posicionamento destes instrumentos entre o mento e o ombro esquerdo requer uma atividade muscular e craniocervical prolongada que, na maioria das vezes, ultrapassa a função fisiológica normal, sendo capaz de gerar uma patologia craniocervical e temporomandibular ${ }^{(10)}$. Em estudos anteriores verificou-se que músicos de viola e violino apresentaram sensibilidade à palpação nos músculos esternocleidomastóideo, trapézio e inserção do músculo temporal esquerdo, com o aparecimento de estalo neste lado $^{(11)} \mathrm{e}$ maior incidência de sensibilidade dolorosa em masseter e temporal, quando comparados com a população em geral $^{(12)}$.

Há, ainda, na execução de alguns grupos de instrumentos de sopro, como tuba, saxofone, trombone, flauta, trompete, a necessidade da aplicação de forças ao sistema estomatognático de forma repetitiva; o que gera um estresse mecânico capaz de, muitas vezes, extrapolar a capacidade fisiológica e produzir um microtrauma. O tipo de embocadura do instrumento - copa ou lingueta - é um fator que merece atenção e se refere ao uso da musculatura oromandibular e, em especial, do músculo orbicular da boca, para a produção do sopro por meio do bocal do instrumento ${ }^{(3)}$.

Neste contexto, a distonia por embocadura foi descrita como distúrbio raro de controle motor, por execução de movimentos repetitivos complexos. Tais movimentos foram observados em um estudo com 26 profissionais de sopro com distonia por embocadura, com média de 40 anos de idade, no qual foi observada limitação em alcançar determinadas notas ou alteração no estilo de tocar, tremor no movimento de embocadura, movimentos involuntários de lábios, dificuldade de fechamento da mandíbula ${ }^{(6)}$.

Alguns estudos relacionaram a embocadura do instrumento e problemas com os dentes ou oclusão que resultaram em alterações na qualidade do sopro produzido, reduzindo o desempenho musical ${ }^{(3,6-7)}$.

Em outro estudo que avaliou 47 instrumentistas de sopro que usavam embocadura em forma de copa e 16 instrumentistas que usavam embocadura em forma de lingueta, foram encontradas alterações orodentais $(59,4 \%)$, aspecto esbranquiçado e deformidades dos lábios $\left(82,6 \%\right.$ ), além de cárie $(88,1 \%)^{(4)}$.

Além disso, frequentemente o músico encontra-se exposto a situações de estresse emocional e ansiedade ${ }^{(13)}$, gerados pela autodisciplina e pela competitividade do meio de trabalho, podendo ocasionar o aparecimento de hábitos parafuncionais como ranger e apertar dentes. Estes fatores fazem dos músicos um grupo de indivíduos suscetíveis a apresentar disfunções na articulação temporomandibular, na musculatura orofacial e nos dentes.

Dessa forma, este trabalho teve por objetivo, verificar a prevalência de sinais e sintomas de disfunção temporomandibular em grupos de músicos intérpretes de instrumentos de sopro e de instrumentos de cordas, que praticam a música como profissão.

\section{MÉTODOS}

\section{Amostra}

Participaram desta pesquisa 92 músicos, com idades variando entre 18 e 58 anos, sendo 82 do sexo masculino e dez do feminino. Foram entrevistados 70 músicos intérpretes de instrumentos de sopro, como metais e palhetas (76,09\%), e 22 músicos intérpretes de instrumentos de cordas, como viola e violino $(23,91 \%)$. Todos eram músicos na cidade de Curitiba, pertencentes à Orquestra Sinfônica do Paraná, Banda da Polícia Militar do Paraná e Banda do Exército, que praticam seus instrumentos com regularidade quase todos os dias. O projeto da pesquisa foi apresentado ao Comitê de Ética da Universidade Tuiuti do Paraná e aprovado sob o protocolo de número $075 / 2005$.

\section{Procedimento}

A pesquisa foi realizada em duas etapas. Na primeira, foi realizada uma palestra explicativa sobre disfunção temporomandibular e sobre os sintomas e sinais envolvidos como: cefaléias, presença de ruídos na articulação temporomandibular, dificuldades para a realização dos movimentos mandibulares, cansaço e/ou dor durante a mastigação, desgastes dentários, dor na articulação temporomandibular, presença de zumbido, sensação de plenitude auricular e hábitos orais deletérios como ranger os dentes e apertamento dentário. Após a explanação, solicitou-se que, nos dias seguintes, os músicos estivessem atentos à presença destes sinais, sintomas e hábitos, para que pudessem ser entrevistados.

A segunda etapa foi realizada cerca de dez a 15 dias após a primeira. Nesta, foi realizada uma entrevista, com perguntas relacionadas em um questionário que continha uma parte referente à identificação dos entrevistados; uma outra, contendo questões acerca do tempo envolvido na prática do instrumento e uma última parte, com perguntas referentes à presença de sinais, sintomas de disfunção da articulação temporomandibular e hábitos parafuncionais - ranger os dentes e/ou apertamento dentário ${ }^{(8)}$ que deviam ser considerados não necessariamente enquanto tocavam o instrumento.

A abordagem aos músicos foi feita em seus locais de ensaio: a Orquestra Sinfônica do Paraná, no Teatro Guairá; a Banda da Polícia Militar, no Quartel General da Polícia Militar do Paraná; a Banda do Exército, no Vigésimo Batalhão de Infantaria Blindada.

\section{Análise estatística}

Para a análise dos dados, o grupo de músicos foi separado em dois grupos, sendo um de intérpretes de instrumentos de sopro e o outro de instrumentos de corda. A comparação entre os grupos, relativa aos sinais e sintomas referidos pelos músicos, foi realizada por meio do teste de diferença de proporções, considerando-se o nível de significância de 0,05 (5\%).

\section{RESULTADOS}

As respostas relativas ao tempo que exerciam a profissão 
Tabela 1. Sinais e sintomas de disfunção temporomandibular e valor de p para os grupos intérpretes de instrumentos de sopro e de corda ( $\mathrm{n}=92$ )

\begin{tabular}{|c|c|c|c|c|c|}
\hline Sinais e sintomas & Sopro & $\%$ & Corda & $\%$ & Valor de $p$ \\
\hline Range e/ou aperta os dentes & 29 & 41,42 & 10 & 45,45 & 0,7394 \\
\hline Cefaléia & 26 & 37,14 & 10 & 45,45 & 0,4878 \\
\hline Dores na ATM & 19 & 27,14 & 4 & 18,18 & 0,3995 \\
\hline Dificuldade para abrir a boca com maior amplitude & 15 & 21,42 & 4 & 18,18 & 0,7609 \\
\hline Escuta ruídos na ATM & 27 & 38,57 & 12 & 54,54 & 0,1895 \\
\hline Presença de zumbido & 26 & 37,14 & 7 & 31,81 & 0,6504 \\
\hline Sensação de plenitude auricular & 30 & 42,85 & 7 & 31,81 & 0,3594 \\
\hline Sente estresse/ansiedade relativos a profissão & 43 & 61,42 & 16 & 72,72 & 0,3377 \\
\hline
\end{tabular}

Teste de diferença de proporções $\mathrm{p}<0,05$

Legenda: ATM = articulação temporomandibular

variaram entre cinco e 40 anos (média de 20,54 anos), e de uma a dez horas, ao tempo de execução diária (média de 4,87 horas por músico). A frequência semanal de apresentações teve uma média de 3,91, variando de uma a dez apresentações por semana.

As respostas referentes à presença de sinais, sintomas e hábitos relacionados às disfunções temporomandibulares, bem como o resultado do teste de diferença de proporções para os dois grupos estão apresentados na Tabela 1.

Considerando-se o nível de significância de 0,05 (5\%), verificou-se que não houve diferença estatisticamente significante entre as proporções de presença de sinais e sintomas entre os instrumentistas de sopro e de corda, pois em todos os casos encontrou-se $\mathrm{p}>0,05$.

\section{DISCUSSÃO}

Apesar de não haver diferença estatisticamente significante entre as ocorrências de sinais e sintomas entre os instrumentistas de sopro e de corda, cabe ressaltar que a incidência de sinais e sintomas em ambos os grupos estudados é alta.

Do total de 39 (42,3\%) músicos que relataram perceber que rangem e/ou apertam os dentes (Tabela 1), cinco músicos que são instrumentistas de cordas, viola e violino, relataram a presença deste hábito enquanto estão tocando o instrumento. Observações acerca deste hábito em músicos instrumentistas de cordas revelaram que, enquanto os mesmos tocavam, apresentavam um desvio da mandíbula para a esquerda, assumindo uma posição de mordida cruzada para este mesmo lado ${ }^{(14)}$. O violino e a viola são posicionados entre o ombro esquerdo e a borda inferior da mandíbula e, muitas vezes, os dentes são mantidos em oclusão para estabilizar a mandíbula e impedir sua deflexão para a direita. Dois músicos relataram ranger os dentes enquanto ouvem música, marcando inconscientemente os tempos das melodias. Não foram encontrados estudos sobre este achado.

Muitos músicos assumem que a sua condição de dor é normal e encontram meios de mascarar os efeitos deste proble$\mathrm{ma}^{(15)}$. Em um estudo com 31 adolescentes violinistas, quando os dados obtidos foram comparados a um grupo controle, não houve diferença referente à frequência das cefaléias, sendo encontrados relatos de cefaléias semanais em $25 \%$ e $22 \%$, nos grupos de estudo e controle, respectivamente. Contudo, crises severas ocasionais foram reportadas por $35 \%$ e $9,6 \%$ nos grupos de estudo e controle, respectivamente ${ }^{(16)}$. No presente estudo, encontrou-se um músico trompista que teve que abandonar a profissão por três anos devido a severos episódios de cefaléia. A frequência das crises de cefaléia em 36 músicos $(39,1 \%)$ variou de uma ao mês a crises diárias e não foram encontradas diferenças estatisticamente significantes entre os grupos de sopro e de cordas (Tabela 1).

Vinte e três $(25 \%)$ músicos, sendo $19(27,14 \%)$ e $4(18,18 \%)$ intérpretes de instrumentos de sopro e corda, respectivamente, disseram sentir dor na articulação temporomandibular, especialmente quando precisavam tocar por longos períodos. Da mesma forma, 19 (20,6\%) músicos de ambos os grupos relataram dificuldade para abrir a boca com maior amplitude (Tabela 1). Estudo semelhante com músicos violinistas adolescentes revelou dor na articulação temporomandibular em abertura bucal máxima em uma relação de $25,8 \%$ e 6,4\% nos grupos de estudo e controle, respectivamente ${ }^{(16)}$. Foi encontrado na literatura especializada, relato de caso de um músico trompista que passou a apresentar dores na articulação temporomandibular e dificuldade para abrir a boca, após seis horas de estudo diário ${ }^{(12)}$. Os tocadores de violas, devido ao maior peso e tamanho deste instrumento, podem sofrer de cefaléias, rigidez no pescoço e dores na região da articulação temporomandibular, ao tocarem de forma continuada por três horas ou mais ${ }^{(14)}$.

Dentre aqueles que executam os instrumentos de sopro, dez músicos $(45,4 \%)$ relataram a presença de dor nos lábios durante ou após tocar o instrumento. Estudo anterior relatou o caso de trompetista com ruptura do músculo orbicular da boca, cujo pai, também trompetista, teve que abandonar a profissão devido ao mesmo problema ${ }^{(17)}$. Nesse grupo de metais como trompa, trompete e trombone, a pressão pode alcançar valores de até 500 gramas. Assim, a duração e a direção das forças produzidas por alguns instrumentos musicais podem ocasionar má oclusão ${ }^{(18)}$, sendo que 100 gramas já é um peso suficiente para mover um dente ortodonticamente ${ }^{(12)}$. Em um estudo cefalométrico com trompetistas que apresentavam má oclusão de classe II divisão 1, com retroposição mandibular, foi observado que, para manter o contato dos lábios com o bocal, estes músicos precisavam projetar muito a mandíbula para frente, o que poderia explicar a presença de sintomas na articulação temporomandibular, devido à fadiga do músculo pterigóideo lateral $^{(19)}$. 
Quanto à presença de ruídos articulares, 39 (42,3\%) músicos relataram presença de ruídos na articulação temporomandibular, sendo que $27(38,57 \%)$ pertencem ao grupo dos instrumentistas de sopro e $12(54,54 \%)$ são instrumentistas de corda (Tabela 1). Em um grupo de 150 músicos profissionais instrumentistas de sopro, foi encontrada, ao exame clínico, alta incidência de estalo e crepitação na articulação temporomandibular de intérpretes de trombone e tuba (31\%) quando comparada ao grupo controle (12\%). Dessa forma, foi demonstrado, por meio de análises cefalométricas e teleradiografias laterais que, ao interpretar a tuba e o trombone, a mandíbula se desloca de uma posição de repouso para cima e para trás de forma repetitiva, favorecendo o deslocamento posterior do côndilo mandibular e aumentando a probabilidade de luxação anterior do disco $\operatorname{articular}^{(20)}$. Em outro estudo, a frequência de ruído na articulação temporomandibular foi de $13 \%{ }^{(21)}$.

A partir desses resultados, é importante ressaltar que, ao receberem um músico para atendimento clínico, os profissionais da Fonoaudiologia estejam atentos a realizar uma entrevista inicial que investigue a ação do respectivo instrumento musical sobre o sistema estomatognatico.

Em relação a sintomas auditivos, 33 músicos $(35,8 \%)$ relataram zumbido, sendo que $26(37,14 \%)$ músicos eram intérpretes de instrumentos de sopro e $7(31,81 \%)$ de cordas, não havendo diferença estatisticamente significante deste sintoma entre os grupos. Além disso, a sensação de plenitude auricular foi relatada por $37(40,2 \%)$ músicos que tocam ambos os instrumentos (Tabela 1). Estes achados corroboram com os encontrados em outros estudos, sendo que em um deles foram avaliados 23 músicos da Orquestra Sinfônica da Universidade Estadual de Londrina e 43,4\% apresentaram zumbido ${ }^{(22)} \mathrm{e}$, em outro, $37 \%$ dos músicos avaliados revelaram essa queixa ${ }^{(23)}$.

A elevada ocorrência de queixas auditivas nessa população pode ser explicada por dois fatores principais: exposição a níveis elevados de pressão sonora e presença de disfunção temporomandibular ${ }^{(24-28)}$. Dessa forma, estes devem estar inseridos em um programa de preservação auditiva.

Alem das queixas relativas à audição e alterações na ATM, os dados apresentados na Tabela 1 revelaram que $59(64,1 \%)$ músicos relataram sentir estresse e/ou ansiedade relativos à profissão, dentre eles, um músico referiu sentir "pânico" nas apresentações. O estresse é um importante fator relacionado à profissão do músico ${ }^{(13)}$.

\section{CONCLUSÃO}

A partir dos resultados do presente estudo foi possível concluir que a somatória dos fatores apresentados coloca os intérpretes dos instrumentos de sopro e de corda, como um grupo suscetível a apresentar sinais e sintomas de Disfunção Temporomandibular. Além disso, tal prática pode ser considerada, tanto um fator desencadeante, quanto um fator agravante ou perpetuador de um problema já existente.

\begin{abstract}
Purpose: To verify the prevalence of signs and symptoms of temporomandibular dysfunction in groups of professional musicians, interpreters of brass and string instruments. Methods: Ninety two musicians, with ages varying from 18 to 58 years, participated on the study. Seventy musicians who played brass instruments (76.08\%) and 22 musicians who played string instruments (23.91\%) at the Symphonic Orchestra of Paraná, the Military Police Band or the Army Band were interviewed. The interview included questions regarding identification, time of practice, and presence of signs, symptoms and habits related to temporomandibular dysfunction. The answers were compared using the difference of proportions test. Results: Thirty nine musicians (42.3\%) noticed teeth grinding and/or teeth pressing, 23 (25\%) reported to feel pain at the temporomandibular joint, 39 (42\%) reported to hear noises at the temporomandibular joint, 37 (40\%) reported sensation of auricular plenitude, and 33 (35\%) presented tinnitus. No significant difference was found between brass and string players regarding the presence of signs and symptoms of temporomandibular dysfunction. Conclusion: The sum of factors presented puts players of certain musical instruments as a group susceptible to present signs and symptoms of temporomandibular disorder, including auditory symptoms. Therefore, the practice of these instruments may be considered a trigger, as well as an aggravating or perpetuating factor of a previously existing problem.
\end{abstract}

Keywords: Temporomandibular joint/pathology; Masticatory muscles; Music; Occupational diseases/diagnosis

\title{
REFERÊNCIAS
}

1. Roset-Llobet J, Rosinés-Cubells D, Saló-Orfila JM. Identification of risk factors for musicians in Calalonia (Spain). Med Probl Perform Art. 2000;15(4):167-74.

2. Schmidt JM, Verschuure J, Brocaar MP. Hearing loss in students at a conservatory. Audiology. 1994;33(4):185-94.

3. Papsin BC, Maaske LA, McGrail JS. Orbicularis oris muscle injury in brass players. Laringoscope. 1996;106(6):757-60.

4. Panzza Negrete AO, Castro Núñez JA, Pedraza Castillo LC, Cabrales
Marrugo M. Morbilidad bucodental en músicos de bandas de porro del departamento de Córdoba. Rev Fac Odontol Univ Antioquia. 2000; 12(1):20-6.

5. Kähäri KR, Axelsson A, Hellström PA, Zachau G. Hearing assessment of classical orchestral musicians. Scand Audiol. 2001;30(1):13-23.

6. Frucht SJ, Fahn S, Greene PE, O’Brien C, Gelb M, Truong DD, et al. The natural history of embouchure dystonia. Mov. Disord. 2001;16(5):899-906. 
7. Conceição Maia AW, Correia de Araújo RP. Influencia de los dientes anteriores en el desempeño del instrumentista de viento. Rev Assoc Odontol Argent. 2002;90(1):23-31.

8. Biasotto-Gonzalez DA. Articulação temporomandibular. In: BiasottoGonzalez DA, organizador. Abordagem interdisciplinar das disfunções temporomandibulares. Barueri: Manole; 2005.

9. Zaza C, Farewell VT. Musicians' playing-related musculoskeletal disorders: an examination of risk factors. Am J Ind Med. 1997;32(3):292-300.

10. Alanen PJ, Kirveskari PK. Occupational cervicobrachial disorder and temporomandibular joint dysfunction. Cranio. 1984-1985;3(1):69-72.

11. Bryant GW. Myofascial pain dysfunction and viola playing. Br Dent J. 1989;166(9):335-6

12. Zimmers PL, Gobetti JP. Head and neck lesions commonly found in musicians. J Am Dent Assoc. 1994;125(11):1487-90, 1492-4, 1496.

13. Hirsch JA, McCall WD Jr, Bishop B. Jaw dysfunction in viola and violin players. J Am Dent Assoc. 1982;104(6):838-43.

14. Herman E. Orthodontic aspects of musical instrument selection. Am J Orthod. 1974;65(5):519-30.

15. Robinson D, Zander J. Preventing musculoskeletal injury (MSI) for musicians and dancers: a resource guide. Safety and Health in Arts Production an Entertainment; 2002. Disponível em: http://www.shape. bc.ca/resources/pdf/msi.pdf

16. Kovero O, Könönen M. Signs and symptoms of temporomandibular disorders in adolescent violin players. Acta Odontol Scand. 1996;54(4):271-4.

17. Planas J. Rupture of the orbicularis oris in trumpet players (Satchmo's syndrome). Plast Reconstr Surg. 1982;69(4):690-3.

18. Engelman JA. Measurement of perioral pressures during playing of musical wind instruments. Am J Orthod. 1965;51(11):856-64.
19. Parker J. The Alameda instrumentalist study. Am J Orthod. 1957;43:399415.

20. Gualtieri PA. May Johnny or Janie play the clarinet? The Eastman Study: a report on the orthodontic evaluations of college-level and professional musicians who play brass and woodwind instruments. Am J Orthod. 1979;76(3):260-76.

21. Vincent SD, Lilly GE. Incidence and characterization of temporomandibular joint sounds in adults. J Am Dent Assoc. 1988;116(2):203-6.

22. Marchiori LLM, Melo JJ. Comparação das queixas auditivas com relação à exposição ao ruído em componentes de orquestra sinfônica. Pró-Fono. 2001;13(1):9-12.

23. Laitinen H. Factors affecting the use of hearing protectors among classical music players. Noise Health 2005;7(26):21-9.

24. Early KL, Horstman SW. Noise exposure to musicians during practice. Appl Occup Environ Hyg. 1996;11(9):1149-53.

25. Pascoal MIN, Rapoport A, Chagas JFS, Pascoal MBN, Costa CC, Magna LA. Prevalência dos sintomas otológicos na desordem temporomandibular: estudo de 126 casos. Rev Bras Otorrinolaringol. 2001;67(5 Pt 1):627-33.

26. Mazzeto MO, Nascimento G, Gomes NM. Estudo da prevalência das alterações das funções estomatognáticas em pacientes com disfunção temporomandibular. J Bras Fonoaudiol. 2002;7:140-7.

27. Mendes MH, Koemler LA, Assencio-Ferreira VJ. A prevalência de perda auditiva induzida pelo ruído em músicos de banda instrumental. Rev CEFAC. 2002;4(3):179-85.

28. Jacob LCB, Rabiço TM, Campêlo RM, Aguiar FP, Zeigelboim BS. Sintomas auditivos e análise das emissões otoacústicas evocadas por estímulo transiente em indivíduos portadores de disfunção temporomandibular. Distúrb Comum. 2005;17(2):173-82. 